\title{
Tradiciones discursivas en Twitter: de la novela por entregas a la de folletuit
}

\author{
Paulo Verdín
}

\begin{abstract}
Resumen
En este artículo se aborda la comparación de las tradiciones discursivas decimonónicas, la novela por entregas y el género novela de folletuit, neologismo creado por el escritor mexicano Mauricio Montiel Figueiras para asignar una marca genérica a su proyecto novelístico publicado en Twitter: El hombre de tweed. El análisis se hace a partir de cinco categorías básicas: medio, obra, autor, editor y lector. El estudio de ambas tradiciones se realiza desde la propuesta del lingüista Kabatek (2005) y busca identificar invariantes en el género y sus transformaciones.
\end{abstract}

Palabras clave: Twitter, tradición discursiva, novela por entregas, novela de folletuit.

\section{Discursive TRADITIONS ON TWITTER: FROM SERIAL NOVELS IN PERIODICALS TO THE FOLLETUIT NOVEL}

\begin{abstract}
This article deals with the comparison of nineteenth-century discursive traditions, serial novels and the genre folletuit novel, a neologism created by Mexican writer Mauricio Montiel Figueiras to assign a generic mark to his novel project published on Twitter: The tweed man. The analysis is made from five basic categories: medium, work, author, editor and reader. The study of both traditions is carried out from the proposal of linguist Kabatek (2005) and its objective is to identify invariants in the genre and its transformations.
\end{abstract}

Keywords: Twitter, discursive tradition, serial novel, folletuit novel.

Dol: http://doi.org/10.22201/codeic.16076079e.2020.v21n3.a3 
"Tradiciones discursivas en Twitter: de la novela por entregas a la de folletuit"

Vol. 21, Núm. 3, mayo-junio 2020

Revista Digital Universitaria

\section{Paulo César Verdín Padilla}

pauloverdinp@gmail.com

https://orcid.org/0000-0002-0203-1180

Twitter:@PauloVerdin

Licenciado en Derecho y en Letras Hispánicas por la Universidad de Guadalajara. Maestro en Literatura Mexicana por la misma casa de estudios. Estudiante del Doctorado en Humanidades de la Universidad de Guadalajara donde desarrolla una tesis de posgrado referente a las novelas en Twitter. Coordinador de la editorial de textos breves Effictio Editores. 
Imagen 1. Cuenta en Twitter de El hombre de tweed (@ Elhombredetweed).

Anteriormente el límite era de 140 caracteres.

2 El proyecto se encontraba en la cuenta@Elhombredetweed, la cual estuvo activa desde el año 2011 hasta abril de 2019. Actualmente, se puede consultar el segundo apartado del proyecto novelístico, "El hombre de tweed: la isla", en la Revista de la Universidad de México, núm. 137, julio 2015, disponible en: http://www. revistadelauniversidad.unam. mx/articulo.php?publicacion $=7$ 91\&art $=16725 \& \mathrm{sec}=\mathrm{Creaci} \% \mathrm{C}$ 3\%B3n
La aparición de nuevas tecnologías en los últimos años y la creación de internet han traído como consecuencia que los procesos de lectura y escritura, la escritura creativa y la composición literaria se hayan visto modificados. Este nuevo mundo digitalizado dio lugar a una forma genérica que actualmente se conoce como literatura electrónica, cuyas características más sobresalientes son la utilización de recursos multimedia, la hipertextualidad y la interactividad.

Dentro de la literatura producida en las redes sociales destaca la llamada twitteratura (o tuiteratura en su versión castellanizada), palabra compuesta por la unión de Twitter y literatura y que utiliza esta plataforma virtual como medio de comunicación. Esta red social funciona como un servicio de mensajería pública e instantánea, cuyos mensajes tienen la característica de limitarse a 280 caracteres, ${ }^{1}$ con publicación en tiempo real. Los usuarios que utilizan este recurso para ejercer la escritura creativa entran constantemente en diálogo con las formas canónicas literarias del mundo impreso. Por su naturaleza, en la plataforma se pueden practicar y escribir géneros breves como el aforismo, el haikú, la minificción, entre otros, pero también géneros de mayor extensión como la novela.

La intención de este artículo es analizar el proyecto novelístico, escrito en Twitter entre los años 2011 y 2019, titulado El hombre de tweed (ver imagen 1)2 del escritor jalisciense Mauricio Montiel Figueiras, como parte de una tradición discursiva perteneciente al género decimonónico de la novela por entregas. El objetivo central es identificar los elementos que se preservan de la tradición, los que ya no se usan y sus innovaciones. El marco teórico que se utilizará son las aportaciones de Kabatek (2005) y Jacob y Kabatek (2001) en torno a las tradiciones discursivas y los lineamientos que marca el canon literario para el género según las investigaciones de Ferreas (2013) y Villegas Cedillo (1984).

\section{El hombre de tweed @Elhombredetweed \\ No estoy donde debería estar. [Cuenta personal creada y administrada por Mauricio Montiel Figueiras, escritor mexicano. Instagram: mauricio_montiel_figueiras.] \\ () Necesito un mapa. \\ $\mathcal{Q}$ prosasycosas.blogspot.mx \\ Se unió en marzo de 2011 \\ Q Nació el 03 de junio}

El estudio propuesto versa sobre lo que Kabatek (2005) llama en sentido restringido tradiciones complejas: los géneros. En este caso, los géneros que se abordarán son de índole literario: novela por entregas y novela de folletuit. La 
comparación se efectuará a partir de los siguientes parámetros: medio, obra, autor, editor y lector para posteriormente verificar las invariantes y variantes textuales entre ambos modelos discursivos.

Kabatek (2005) parte del supuesto de que todas las Tradiciones Discursivas (TD) implican:

la repetición de un texto o de una forma textual o de una manera particular de escribir o de hablar que adquiere valor de signo propio [...]. Se puede formar con cualquier finalidad de expresión o con cualquier elemento de contenido cuya repetición establece un lazo entre actualización y tradición [...] (2005, p. 159).

Señala, además, que estas repeticiones pueden ser parciales o totales y que es necesario establecer una relación temporal entre textos de diverso momento histórico. En este sentido, interesa observar la tradición formal entre géneros, es decir, la forma textual novela por entregas y la novela de folletuit para verificar la evolución de estas tD en su aspecto diacrónico.

El folletín y la novela por entregas son modalidades que estuvieron en boga en el siglo xIx. Se definen por el medio de transmisión y los receptores a los que se dirigen (García Berrio y Huerta Calvo, 1995, p. 190). El folletín se publicaba de forma periódica en la parte inferior de un diario, mientras que las entregas estaban constituidas en cuadernillos o fascículos. Sin embargo, con el tiempo, llegaron a identificarse como una sola, designada como novela por entregas. Su antecedente inmediato son las novelas de caballería y la producción de estas obras se explican desde la óptica de una cultura de masas.

Por su parte, la denominación novela de folletuit, creada por Mauricio Montiel Figueiras, da la clave para enlazar su obra a la tradición discursiva de la novela por entregas. El neologismo folletuit es una palabra compuesta, formada a partir de la palabra folletín ${ }^{3}$ y tuit (versión castellanizada de la palabra tweet). Por lo que remite a la forma de publicación temporal propia de los folletines, alude sinecdócticamente a la plataforma Twitter y resalta que la novela en construcción presenta uno de sus rasgos característicos: publicación de mensajes instantáneos de $140^{4}$ caracteres.

\section{Medio}

La denominación genérica de ambas tradiciones discursivas alude al medio donde se transmiten. En este tipo de obras predomina el continente y no el contenido. Además, el medio condiciona fuertemente la escritura de las obras.

${ }^{3}$ La palabra, al igual que el género tienen su origen en Francia de feuilleton, diminutivo de feuillet, hoja, página de un

libro.

${ }^{4}$ Fue un rasgo definitorio hasta casi finales del año 2017. Wolfgang Raible (1988) lo señala como una de las seis dimensiones de la que emanan los rasgos del que se sirve el género.

En el siglo xix el periodismo cobra una vital importancia, ya que es en periódicos y revistas donde las novelas por entregas fueron publicadas. La doctora en Letras Modernas, Marie-Eve Thérenty en una entrevista (García, 
2012) afirma que este tipo de prensa sólo debe pensarse entre dos aspectos: el de la opinión y el de la información. Pero especifica, que en el terreno de la literatura, su aportación fue fundamental pues "toda obra (novela, teatro, poesía) se publicó primero en la prensa y después se convirtió en libro, [agrega, además, que] todos los escritores, salvo contadas excepciones, fueron periodistas;" (p. 8) y que la diferencia de la prensa decimonónica con la contemporánea radica en "la hibridación literaria; [que] hoy no tiene cabida" (p 13).

Por otra parte, Twitter es un servicio de mensajería pública e instantánea de contenidos breves, limitados a un cierto número de caracteres, 280 actualmente. En este sentido, la plataforma, hospedada en el sitito web de internet twitter. com, no tiene una finalidad definida, su idea rectora es dejar que las personas cuenten lo que sucede, tal y como lo indica la pregunta "¿Qué está pasando?", que aparece siempre en un rectángulo ubicado en la parte superior de la pantalla para redactar tuits y que interpela constantemente a los usuarios. ${ }^{5}$

Desde esta ausencia de finalidad específica, el escritor Montiel Figueiras, ha utilizado este medio digital con fines artísticos para ir creando su proyecto narrativo en la cuenta @Elhombredetweed. El modo de almacenamiento de la obra, por tanto, es la red, es decir, de manera online; y al tratarse de un proyecto inacabado se presenta como una versión intermedia. Esta modalidad es importante porque hace de la obra una materia fugaz, fractal, seriada e híbrida, semejante a la novela por entregas.

\section{Obra}

Ferreas (2013) comenta que las novelas por entregas en el siglo xIx eran equiparables a una mercancía, sobre todo por el ámbito comercial que las rodeaba. Se regían bajo las leyes del mercado: duración y extensión, además de ser fabricadas en serie y, por tanto, el autor se asemejaba a un obrero de la literatura.

En cuanto a los aspectos formales que presentaban los textos literarios, éstos se distinguían por utilizar papel de mala calidad y usar caracteres de imprenta demasiado grandes. Las entregas se conformaban más o menos por 16 páginas de 20 a 25 líneas. Comúnmente oscilaban entre 52 y 100 entregas que juntas formaban dos tomos con 400 páginas cada una. Villegas Cedillo (1984), por su parte, asienta que las características tipográficas del género están destinadas a un lector nuevo e inexperto. La división en capítulos, y éstos en apartados menores, generaba, además, enormes ganancias para los editores, ya que se podía jugar con la extensión de la obra, pues permitía llenar al autor páginas y páginas.

La estructura de estas obras estaba predeterminada, tenía un lenguaje característico con un estilo llano y sencillo. Los personajes que intervenían en las historias eran estereotípicos, es decir, representaban los modelos de la época y 
la problemática estaba dada de antemano. En cuanto al tema, muchas veces era dado por el editor. Villegas Cedillo (1984) anuncia algunas de sus características: preferencia por lo extraordinario, menosprecio de la realidad, agitación de sentimientos, adjetivación efectista, fantasía, tensión, barroquismo, sorpresa.

Por otra parte, la novela de El hombre de tweed, por estar constituida en un soporte digital, presenta características diferentes. La obra no está organizada en página sino en tuits. Entre ellos, muchos fungen a manera de índice o como aparato de lectura, pues especifican, por ejemplo, el número de capítulo y nombre del apartado al que pertenece la secuencia (ver imagen 2).

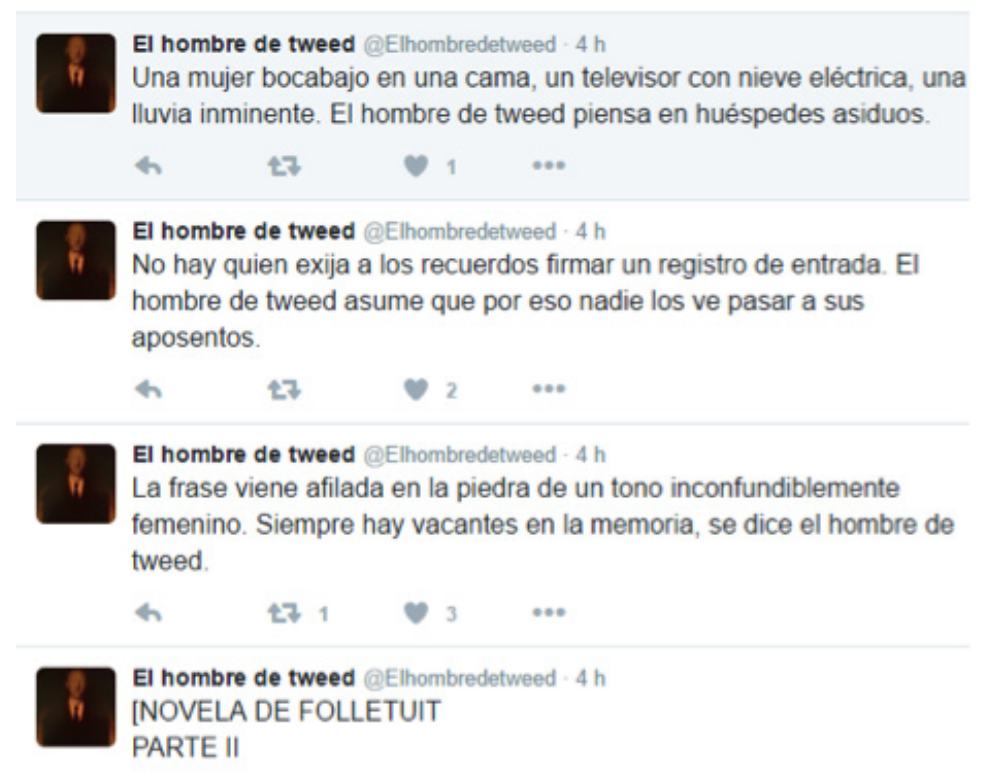

Imagen 2. Tuits de la novela El hombre de tweed (@ Elhombredetweed).

${ }^{6}$ La fuente Arial fue diseñada en 1982 específicamente para tener una mejor resolución en pantallas e impresoras. Sus creadores fueron Roben Nicholas y Patricia Saunders (Ferrer González y Gómez Fontanills, 2011).

${ }^{7}$ Mauricio Montiel ha escrito tres entregas de la novela con el límite de los 140 caracteres. Actualmente Twitter permite la escritura de 280 caracteres.

${ }^{8}$ Estos signos también aparecen en lo tuits que organizan la novela en capítulos y apartados.
"EL HOMBRE DE TWEED:
LA ISLA"

CAPITULO 12]

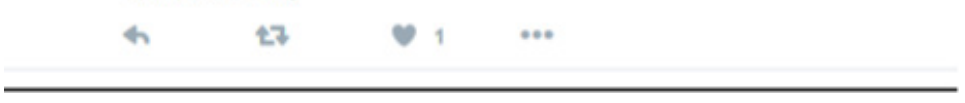

La tipografía está determinada por la empresa Twitter. La letra Arial ${ }^{6}$ y el tamaño 10.5 son los tipos digitales que se utilizan como fuente a la hora de la escritura. Otra limitante que tiene el escritor-editor en este aspecto es que no puede usar otros recursos tipográficos como negritas o cursivas. Los párrafos están acotados a 140 caracteres $^{7}$ y el leer la secuencia novelesca, conformada por conexiones entre un tuit y otro, se hace con una direccionalidad inversa, es decir, de abajo hacia arriba.

El autor ha creado sus propias normas para darle un orden al sistema de lectura. Por ejemplo, siempre publica entre corchetes todo aquel contenido que no forma parte de la narrativa de la novela. ${ }^{8}$ Los corchetes ayudan a distinguir 
entre el autor y la obra. Así estos signos tipográficos se presentan en su sentido originario como una inserción complementaria o aclaratoria que indican la voz del autor que comparte la cuenta al mismo tiempo con el protagonista de la novela. Hay que destacar que la novela es de acceso abierto y no tiene fin de lucro directamente. Sin embargo, la plataforma sí es usada con fines comerciales, pues la empresa Twitter publicita anuncios en las cuentas de sus usuarios.

\section{Autor y editor}

Las novelas por entregas son definidas ante todo como un modo editorial, un modelo de negocio en el que participan lectores, autores y editores. El escritor en este caso es contratado por el editor por una remuneración semanal. En su contrato se estipula como obligación principal la entrega de una cantidad determinada de páginas semanalmente. El éxito de algunas novelas por entregas y el ritmo de trabajo los obligaba muchas veces a dictar sus obras a un taquígrafo en lugar de escribirlas. Cabe mencionar que estos autores escribían por entregas, pero no tenían una obra completa que iban entregando al editor, sino que la mayoría de las veces no existía ni siquiera un plan previo.

El editor, en cambio, coincide con la figura del empresario, pues en sus funciones recaen las actividades de impresión, edición y difusión de las obras. Es quien busca y contrata a los escritores por entregas mediante una remuneración económica. Dentro de sus facultades principales se encontraba la capacidad de decidir el período de duración de la entrega y, de esa forma, podía acortar o extender el plazo según el éxito o fracaso de la obra.

Dentro de la novela de folletuitel modelo comercial no se encuentra presente. La figura de autor y editor aparecen fusionadas. No existe un intermediario quien contrate ni difunda la obra. El mismo autor es quien escribe, revisa, pule, publica y edita sus textos e incluso puede retirarlos de la timeline si así lo desea pues es una posibilidad que brinda la plataforma. En la publicación de la obra no existe un beneficio directo económico para el autor, aunque la cuestión comercial no es ajena a la plataforma. La escritura aparece entre anuncios que se muestran continuamente en la timeline, además de que el autor utiliza su cuenta para la autopromoción de su imagen como escritor, sus publicaciones y talleres. Es también quien determina la duración de las entregas y la manera de publicación de la novela. Asimismo, establece un contacto con sus lectores mediante tuits anunciando la hora y el día en que se irán publicando los folletuits y las entregas.

\section{Lectores}

Una característica que tiene en común la novela por entregas y la novela de folletuit es que el público al que van dirigidos es un sector delimitado. En el caso de la novela por entregas, los lectores coincidían con los consumidores del diario o revista en que se publicaba el folletín correspondiente. Ferreas (2013), 
en su estudio sobre la novela en el siglo xix en España, señala que estas obras eran dirigidas a una esfera social económicamente débil, principalmente a la clase obrera, al pequeño burgués y al público femenino. Por su parte, Cedillo Villegas (1984) indica que en México la novela por entregas permeó todas las capas sociales.

Los lectores de este tipo de novelas tenían la oportunidad de coleccionar los folletines y fascículos, luego llevarlos a un taller de encuadernación para darles forma de libro. Villegas Cedillo (1984) apunta que los editores, para granjearse al público, le regalaban, entre otras cosas, cubiertas y grabados. Los suscriptores posteriormente llevaban a los talleres de encuadernación estos materiales para formar un libro con los fascículos coleccionados. Este gasto desde luego corría a cargo de los lectores.

La comunicación con los receptores de las obras se hacía fundamentalmente mediante la prensa, carteles y boletines en la librería. Por ejemplo, Manuel Payno anunció en 1871, en el periódico El Federalista, la próxima publicación en folletín del segundo tomo de su novela El hombre de la situación.

La novela folletuit, por su parte, también tiene un público acotado que se delimita por el medio. Los lectores tienen que contar con acceso a internet, ser usuarios de la plataforma Twitter y seguir la cuenta donde se publica la novela. Los lectores, en este caso, son identificados con los seguidores o followers de la cuenta @elhombredetweed y pueden ser de cualquier parte del mundo, donde exista conectividad a la red. En este sentido, hace falta determinar un estudio sobre los parámetros sociales de los lectores que siguen la historia: edad, sexo, clase, educación, nacionalidad, entre otros. En 2017 la cuenta contaba con más de 19,000 seguidores.

En cuanto a la situación comunicativa, ésta se presenta de manera directa. Chimal (2014), en su estudio sobre la tuiteratura, apunta la interacción instantánea y diversa como una de las características de este fenómeno literario. La red permite publicar enlaces (mensajes con hipervínculos), usar etiquetas (hashtags), que indican un determinado tema o evento, ofrecer respuestas y réplicas en conversaciones, enviar mensajes directos entre cuentas, retuitear (reenvíos de tuits), marcar como favorito algún contenido, etcétera. Incluso, la red permite crear pequeñas encuestas donde también se puede inferir la aprobación y los gustos de los seguidores. Todas esas herramientas virtuales posibilitan una comunicación inmediata y continua entre autor y lectores.

\section{A modo de conclusión}

Jacob y Kabatek (2001) afirman que las "las tradiciones discursivas, en tanto formas históricas, están sometidas al cambio" (p. 207). La evolución de las tecnologías en este sentido ha afectado las formas de escritura y lectura. La novela por entregas y la novela de folletuit, si bien pertenecen a períodos históricos distintos, 
guardan una cierta relación en cuanto a sus modelos discursivos.

Algunos rasgos específicos que comparten como características invariantes son las siguientes: a) nombre y definición genérica dada por el medio en el cual se originan; b) la temporalidad como un elemento clave para dar a conocer la historia; c) la fragmentación de la obra; d) la producción de sentido en forma seriada; e) el uso estratégico del suspenso para mantener cautivo al lector; f) la falta de plan previo y proceso continuo de creación de la obra; g) la gran extensión y h) la nota distintiva de la novela, la narrativa.

Hasta aquí se indica una actualización de la tradición discursiva de la novela por entregas; sin embargo, algunos rasgos específicos no se ven modificados. La novela de folletuit, por ejemplo, no se basaba en el modelo de negocio que originó la novela por entregas, sino que consistía en un modelo de acceso abierto a la obra. ${ }^{9}$ Asimismo, la figura del editor se transforma, ya no es identificada con el empresario sino con el autor, debido a que la red da la posibilidad a los creadores de autopublicarse sin intermediarios.

La comunicación, si bien es cierto, existe en ambas tradiciones discursivas, las publicaciones literarias en Twitter se distancian de las novelas por entregas en el sentido de que la plataforma hace posible una comunicación instantánea entre autor y lector. Por último, el soporte impreso es cambiado por el electrónico, lo que conlleva cambios en la tipografía y en los procesos de escritura y lectura: lectura vertical en orden inverso, interacción instantánea y diversa, yuxtaposición de los tuits de la cuenta con tuits de otros usuarios en la línea de tiempo, fugacidad de contenidos y escritura en tiempo real. Finalmente, Mauricio Montiel Figueiras al rescatar una tradición cultivada por Balzac en el siglo xix le ha dado un giro inesperado no sólo al género sino también al uso de la red social.

${ }^{9}$ Al menos inicialmente, porque Mauricio Montiel Figueiras ha manifestado su intención de dar a conocer la última entrega en un libro impreso conjuntamente con las demás, razón por la que tal vez ha eliminado la obra en línea y podría entrar en el esquema editorial tradicional.

\section{Referencias}

* Chimal, A. (2014). De Tuiteratura. Plesiosaurio. Primera revista de ficción breve peruana, 1(7), 157-161.

* Ferreas, Juan Ignacio. (2013). La novela en el sig/o xIx. ACVF Editorial.

* Ferrer González, Alba y Gómez Fontanills, David. (2011). Escritura y tipografía [colección voc]. Titivillus.

* García Berrio, Antonio y Huerta Calvo, Javier. (1995). Los géneros literarios: sistema e historia. Cátedra.

* García, Jaimeduardo. (2012, 29 de enero). El siglo xix, inicio de la era mediática. Jornada Semanal, núm. 882. https://www.jornada.com.mx/2012/01/29/sem-jaime.html.

* Jacob, Daniel y Kabatek, Johannes (Eds.) (2001). Lengua medieval y tradiciones 
discursivas en la Península Ibérica. Descripción gramatical - pragmática histórica metodología. Vervuert:Iberoamericana, 199-231.

- Kabatek, Johannes (2005). Tradiciones discursivas y cambio lingüístico. Lexis, 29 (2), 151-163.

- Montiel Figueiras, Mauricio [@Elhombredetweed]. (2011-2019). El hombre de tweed. Twitter. https://twitter.com/Elhombredetweed [cuenta eliminada].

* Orihuela, José Luis (2011). Mundo Twitter. Alienta editorial.

* Raible, Wolfgang. (1988). ¿Qué son los géneros? En Miguel A. Garrido Gallardo (Ed.), Teoría de los géneros literarios. Arco Libros.

- Villegas Cedillo, Alberto (1984). La novela popular mexicana en el sig/o xIx. Universidad Autónoma de Nuevo León. http://cd.dgb.uanl.mx/handle/201504211/7270.

\section{Cómo citar el artículo}

* Verdín, Paulo (2020). Tradiciones discursivas en Twitter: de la novela por entregas a la de folletuit. Revista Digital Universitaria (RDU) Vol. 21, núm. 3 mayo-junio. Dol: http://doi.org/10.22201/codeic.16076079e.2020.v21n3.a3.

Recepción: 05/07/2018. Aceptación: 11/12/2019. 